\title{
Neues Nomogramm für die Prostatakrebsmortalität
}

\begin{abstract}
Wie hoch ist das Risiko, nach einer radikalen Prostatektomie an Prostatakrebs zu sterben? Diese Frage war bislang kaum zu beantworten. Statistiker und Urologen verschiedener US-amerikanischer Universitäten haben sich gemeinsam auf die Suche nach einer Antwort gemacht.
\end{abstract}

$\mathrm{D}$ ie Zeit, bis ein durch Screening entdeckter Prostatakrebs auch ohne Screening auffällig geworden wäre, beträgt schätzungsweise 11 Jahre. Diese außergewöhnlich lange „lead time“ zeigt den generell langsamen Verlauf der Erkrankung und erklärt, warum in klinischen Studien der PSA-Wert und nicht die Prostatakrebs-spezifische Mortalität (PCSM) als Endpunkt gewählt wird. Spezielle Modelle (Nomogramme) mit denen sich der Wiederanstieg des PSA-Wertes vorhersagen lässt, sind die wichtigsten Instrumente, um Prostatakrebspatienten bei der Therapiewahl zu beraten.

Allerdings wird dabei leicht vergessen, dass der PSA-Wiederanstieg kein Surrogat für die PCSM ist. Innerhalb von 15 Jahren nach einem PSA-Wiederanstieg ist das Risiko, an Prostatakrebs zu sterben, genauso hoch wie das Risiko, aus anderen Gründen zu versterben.

Um ein exakteres Nomogramm zu entwickeln, analysierten Andrew Stephenson und Kollegen das PCSM-Risiko von über 12.000 Patienten, die sich zwischen 1987 und 2005 einer radikalen Prostatektomie unterzogen hatten. Die

\section{Nomogramm zur Bestimmung der Prostatakrebs-spezifischen $\quad$ Grafik 1 10- bzw. 15-Jahres-Mortalität}

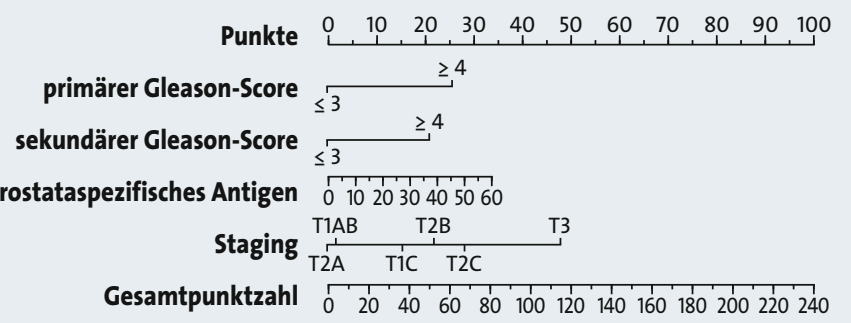

prostatakrebsspezifische 10-Jahres-Mortalitätsrate prostatakrebsspezifische 15-Jahres-Mortalitätsrate

\begin{tabular}{|c|c|}
\hline $0,010,02$ & 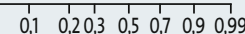 \\
\hline
\end{tabular}

Primären Gleason-Score eintragen, senkrechte Linie zur Punkteskala ziehen, um die Punktzahl zu bestimmen; das Verfahren für die weiteren drei Parameter wiederholen. Gesamtpunktzahl in die vorgesehene Skala eintragen, senkrechte Linie nach unten ziehen und 10- bzw. 15-Jahres-Mortalität bestimmen.

15-Jahre-PCSM betrug 12\%, die Gesamtmortalität $38 \%$. Der Gleason-Score, PSA und das Operationsjahr waren mit dem PCSM assoziiert. Mithilfe eines daraufhin entwickelten Nomogramms ließ sich das 15-Jahre-PCSM-Risiko berechnen (Konkordanzwert: 0,82). Weder die präoperative PSA-Geschwindigkeit noch der BMI verbesserten die Genauigkeit

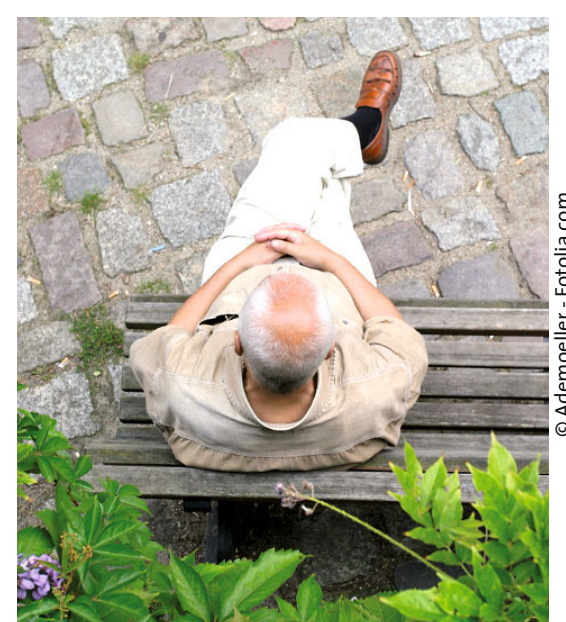

des Modells. Nur 4\% der aktuell behandelten Patienten wiesen aufgrund des Nomogramms eine 15-Jahre-PCSM von über $5 \%$ auf.

Fazit: Nur sehr wenige Patienten werden in den 15 Jahren nach einer radikalen Prostatektomie sterben. Mithilfe der jetzigen klinischen Parameter ist es kaum möglich, Patienten mit einem wesentlich erhöhten Mortalitätsrisiko ausfindig zu machen. Es ist daher dringend notwendig, neue Marker zu finden, die zwischen lebensgefährlichen und harmlosen Prostatatumoren unterscheiden können. fah

Stephenson AJ et al. Prostate cancerspecific mortality after radical prostatectomy for patients treated in the prostatespecific antigen era. J Clin Oncol 2009, 27 4300-5. 